\title{
Resting state fMRI in the moving fetus: a robust framework for motion, bias field and spin history correction
}

\author{
Giulio Ferrazzi ${ }^{\mathrm{a}, 1, *}$, Maria Kuklisova Murgasova ${ }^{\mathrm{a}}$, Tomoki Arichia ${ }^{\mathrm{a}, \mathrm{b}}$, \\ Christina Malamateniou ${ }^{\mathrm{a}}$, Matthew J. Fox ${ }^{\mathrm{a}}$, Antonios Makropoulos ${ }^{\mathrm{a}}$, \\ Joanna Allsop ${ }^{\mathrm{a}}$, Mary Rutherford ${ }^{\mathrm{a}}$, Shaihan Malik ${ }^{\mathrm{a}}$, Paul Aljabar ${ }^{\mathrm{a}}$, Joseph \\ V. Hajnal ${ }^{\mathrm{a}}$ \\ ${ }^{a}$ Centre for the Developing Brain, Division of Imaging Sciences 63 Biomedical \\ Engineering, King's College London, St Thomas' Hospital, Westminster Bridge Rd \\ London SE1 ' 'EH, UK \\ ${ }^{b}$ Department of Biomedical Engineering, Imperial College London, South Kensington \\ Campus London SW7 2AZ, UK
}

\begin{abstract}
There is growing interest in exploring fetal functional brain development, particularly with Resting State fMRI. However, during a typical fMRI acquisition, the womb moves due to maternal respiration and the fetus may perform large-scale and unpredictable movements. Conventional fMRI processing pipelines, which assume that brain movements are infrequent or at least small, are not suitable. Previous published studies have tackled this problem by adopting conventional methods and discarding as much as $40 \%$ or more of the acquired data.

In this work, we developed and tested a processing framework for fetal Resting State fMRI, capable of correcting gross motion. The method comprises bias field and spin history corrections in the scanner frame of reference, combined with slice to volume registration and scattered data interpolation to place all data into a consistent anatomical space. The aim is to recover an ordered set of samples suitable for further analysis using standard tools such as Group Independent Component Analysis (Group ICA).
\end{abstract}

\footnotetext{
*Corresponding author

Email address: giulio.ferrazzi@kcl.ac.uk (Giulio Ferrazzi)

${ }^{1}$ Corresponding author's contact number: +44 7447968570
} 
We have tested the approach using simulations and in vivo data acquired at 1.5T. After full motion correction, Group ICA performed on a population of 8 fetuses extracted 20 networks, 6 of which were identified as matching those previously observed in preterm babies.

Keywords: Fetal fMRI, Slice to volume registration, Resting state networks, Scattered interpolation, Bias field correction, Spin history correction

\section{Introduction}

Resting State Networks (RSNs) are consistently mapped in different human populations with functional MRI (fMRI) and are the topic of extensive Neuroscience research (Van Den Heuvel and Hulshoff Pol, 2010). RSNs are characterized by low frequency temporal fluctuations of the blood-oxygen-leveldependent (BOLD) signal which are correlated between distinct anatomical regions when a subject is imaged at rest, or at least with no prescribed activity or stimulus (Biswal et al., 1995; Buckner et al., 2008). Spatial mapping of RSNs in adults suggests that involved areas are functionally relevant, and include regions involved in motor, visual, auditory, executive and memory functioning (Damoiseaux et al., 2006). RSNs have also been described in infants, and include many of those regions seen in the mature brain (Gao et al., 2009; Damaraju et al., 2010; Fransson et al., 2007, 2009, 2011).

The emergence of RSNs during the preterm period was explored by Smyser et al. (2010) and Doria et al. (2010), who were able to map a full repertoire of networks encompassing the visual, auditory, somatosensory, motor, and executive control areas. These infant studies have identified a maturational trend during this period (equivalent to the third trimester of gestation) consisting of a progression from lateralized networks to bilateral patterns at full term equivalent post-menstrual age (PMA).

The studies on preterm babies suggest that it is of great interest to look at the development of RSNs directly in utero. However, this is a challenge, as during the several minutes taken to acquire a typical fMRI dataset, the fetal brain undergoes large-scale motion caused by changes in fetal position within the womb and also as a result of maternal respiration.

Fetuses move sporadically and in an unpredictable fashion (Malamateniou et al., 2013). These movements take place in both the in-plane and the outof-plane directions. Motion, particularly head rotations and nodding, can be 
rapid. The quality and duration of periods of motion varies with gestational age, such that, as the fetuses get larger later in pregnancy, they may have longer quiet periods and body motion is more constrained (Hayat et al., 2011).

Some preliminary studies have explored fetal RSNs using conventional processing pipelines. Schöpf et al. (2012) acquired fMRI data from 87 healthy fetuses and used single subject Probabilistic ICA (Beckmann and Smith, 2004) to identify RSNs. However, because of large motion levels, 71 datasets couldn't be processed. Thomason et al. (2013) analysed a set of 25 healthy subjects in the second and third trimester of gestation using Group ICA (Calhoun et al., 2009) and correlation analysis. In this study, individual time frames were rejected, with single volumes removed for the time series when there was judged to be excessive motion. The reported rejection rate was $41.28 \%$.

Figure 1 illustrates an example of changes in fetal position for a 28 week old fetus over a period of 4 minutes. The Figure shows 3 images taken from a realtime balanced steady state free precession (bSSFP) cine sequence acquired at 3.3 frames per second. The fetal head position changes in a complex series of 3 dimensional movements that cause the anatomical content within a slice at a fixed location in the scanner to change substantially. Within any single stack of slices that provide whole brain coverage the fetus may therefore change position significantly, so that the conventional approach of correcting motion by realigning whole imaging volumes is likely to result in registration errors (Kim et al., 1999).

This work proposes a framework for processing fetal fMRI data that is designed to operate when there is large-scale and frequent movement. The approach seeks to directly accommodate movement on a slice-by-slice timescale and to correct for signal variations due to changes in position of the fetal head with respect to a spatially varying receiver coil sensitivity distribution. A consequence of fetal motion is that anatomical locations are sampled at irregular time intervals introducing spin history effects that are likely to be variable (Bhagalia and Kim, 2008; Yancey et al., 2011). The proposed method seeks to model and correct for these, before rendering the now scattered data samples back onto a regular coordinate system, enabling further analysis by standard methods such as Group ICA. The aim is to achieve a robust framework that allows as much as possible, ideally all, of the acquired data to be retained and used as part of the Resting State Network analysis. 


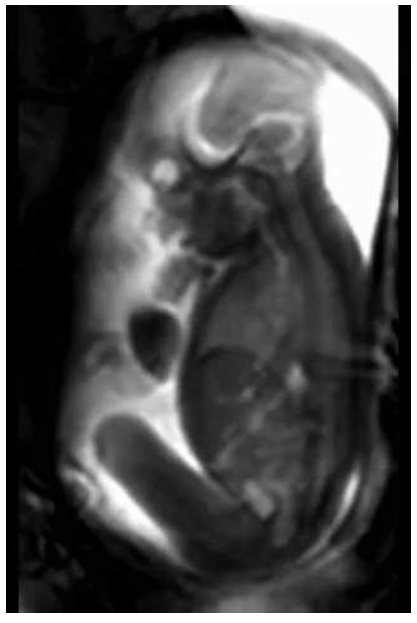

(a) Frame 1.

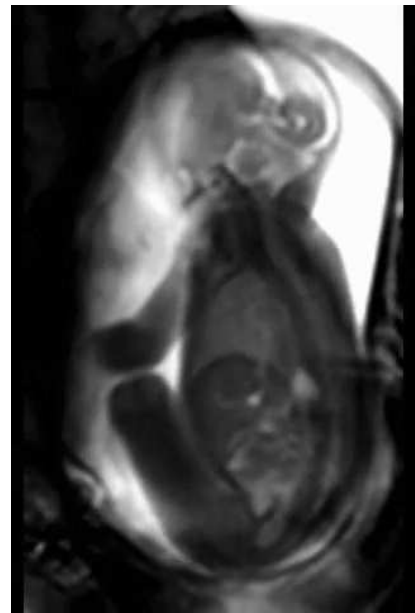

(b) Frame 2.

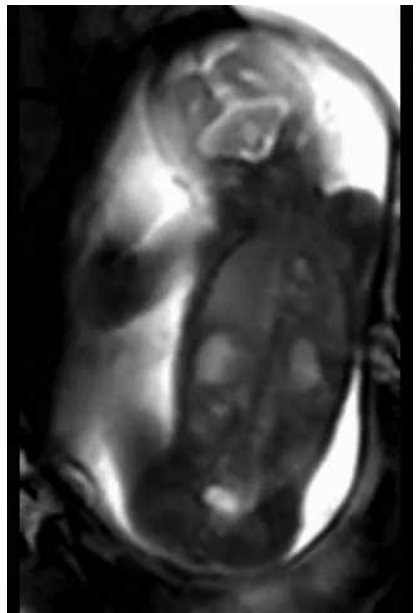

(c) Frame 3.

Figure 1: Three different frames taken from a real time cine MR sequence showing significant motion in a fetal subject at 28 weeks of gestation. The frames were extracted approximately at 18, 36 and $90 s$ from the beginning of the acquisition.

\section{Materials and Methods}

\subsection{Acquisition}

The data used in this study were from 16 fetuses (mean gestational age: $30.37 \pm 4.35$ weeks) who had been assessed as normal and were scanned on a Philips Achieva $1.5 \mathrm{~T}$ scanner with a 32 channel receiver coil using single shot EPI $\left(T_{R}=4000 \mathrm{~ms}, T_{E}=50 \mathrm{~ms}\right.$ with an in-plane resolution of $2.5 \cdot 2.5$ $\mathrm{mm}^{2}$ and slice thickness of $5 \mathrm{~mm}$ ). During each $T_{R}$ interval, 35 slices were

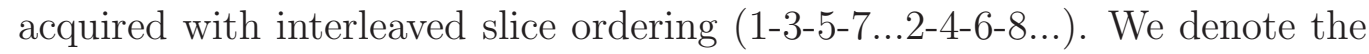
time to acquire a single slice as $T_{S}$.

To make the sampling as dense as possible, the slice positions were overlapped by up to $2.5 \mathrm{~mm}$, with the overlap selected to ensure a large enough stack volume to encompass the fetal brain with a margin for motion.

A SENSE factor of 2 was used for all EPI acquisitions with calibration scans obtained at the beginning of the examination. Since the receiver coil used was fixed relative to the maternal anatomy, fetal movements within the womb changed only local anatomical content, with no substantial effects on coil sensitivity maps. The SENSE calibration scans were rerun if the mother changed 
her position substantially with respect to the scanner bore during the examination. This was easily detected as all fetal images also contain substantial information about the maternal anatomy.

A complete fMRI acquisition consisted of 100 volumes acquired in a single dynamic time series. We also acquired a smaller number of volume stacks of slices in the coronal and sagittal planes to assist the registration algorithm by providing spatial information from different orientations. The phase encoding direction for transverse and coronal views was Anterior-Posterior, so that spatial distortions were nominally in the same direction for these acquisitions. The number of acquired frames in the coronal and sagittal directions was chosen depending on the available scanning time. Single shot Fast Spin Echo (ssFSE) images were also acquired in 3 nominally orthogonal anatomical planes for all subjects $\left(T_{R}=15000 \mathrm{~ms}, T_{E}=180 \mathrm{~ms}, 5\right.$ packages with a resolution of $1.25 \cdot 1.25 \mathrm{~mm}^{2}, 2.5 \mathrm{~mm}$ slice thickness overlapped by 1.25 $m m)$.

Functional studies employ prolonged data acquisitions, in this case almost 7 minutes for the transverse view. This is longer than routinely used for purely anatomical imaging, which in our practice consists of multiple shorter acquisitions typically lasting 1-2 minutes each. There is thus an increased risk that fetuses may change position substantially in the womb during an fMRI acquisition. Despite prescribing imaging stacks with larger anatomical coverage than the actual fetal brain dimensions, there were 3 subjects that moved sufficiently to cause part of the brain to be outside the field of view, resulting in unrecoverable data loss (Figure 2a). In a further 2 subjects there was substantial localized signal loss in part of the brain caused by gas bubbles in the maternal gut (see example in Figure 2b). These 5 cases were excluded from this study.

Table 1 summarizes gestational age, slice overlap and the number of frames that were acquired in the coronal and sagittal directions for every subject that was imaged. Those subjects that were excluded because of incomplete data are shaded in blue.

The remaining 11 datasets constituted the study population upon which the methods were tested; in these instances subjects labelled 1-8 could be fully processed, and those labelled 9-11 couldn't be corrected because of excessive motion not recoverable by the registration algorithm. 


\begin{tabular}{|c|c|c|c|c|}
\hline Fetus & Weeks & Overlap $(\mathrm{mm})$ & Frames (Cor, Sag) & Comments \\
\hline 1 & 32 & 1.5 & $10,-$ & Corrected \\
\hline 2 & 34 & 2 & 10,10 & Corrected \\
\hline 3 & 26 & 2.5 & 10,10 & Corrected \\
\hline 4 & 30 & 2.5 & $2,-$ & Corrected \\
\hline 5 & 27 & 2.5 & $10,-$ & Corrected \\
\hline 6 & 38 & 1 & 10,10 & Corrected \\
\hline 7 & 36 & 2.5 & 10,10 & Corrected \\
\hline 8 & 33 & 2 & 10,10 & Corrected \\
\hline 9 & 33 & 1.5 & $20,-$ & Excessive motion \\
\hline 10 & 25 & 2.5 & $20,-$ & Excessive motion \\
\hline 11 & 26 & 2.5 & 20,20 & Excessive motion \\
\hline 12 & 34 & 2.5 & $20,-$ & Out of FOV \\
\hline 13 & 26 & 2.5 & $2,-$ & Out of FOV \\
\hline 14 & 27 & 2.5 & $20,-$ & Out of FOV \\
\hline 15 & 25 & 2.5 &,- 20 & Bubble \\
\hline 16 & 34 & 2.5 & $20,-$ & Bubble \\
\hline
\end{tabular}

Table 1: Fetal gestational age, amount of overlap between slices and number of frames in coronal and sagittal directions.

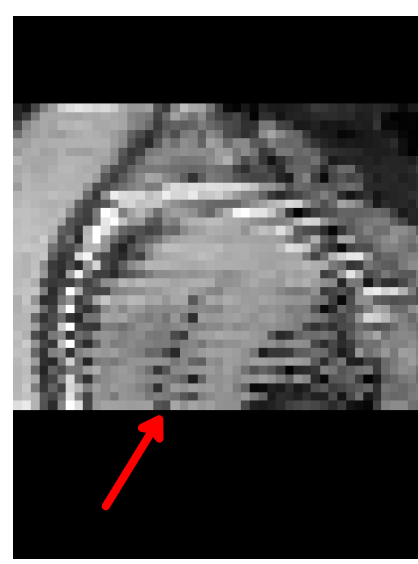

(a) Brain out the field of view.

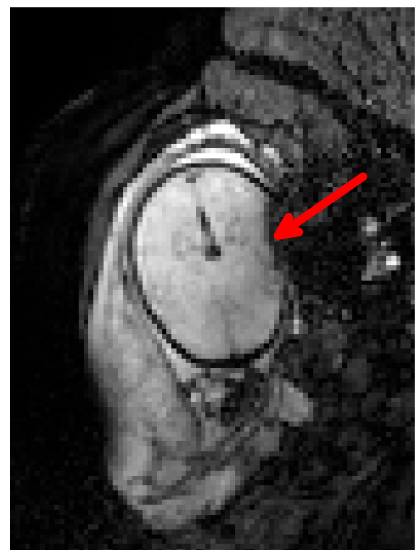

(b) Signal drop due to bubble artefact.

Figure 2: In (a) the fetus moved so that part of the brain was no longer within the imaging volume (red arrow). In (b) a gas bubble in the maternal intestine caused signal loss in the fetal brain (red arrow). 


\subsection{Processing Pipeline}

To process the acquired data we define two distinct coordinate systems: $\mathbf{S}_{1}$ denotes the native scanner space, and $\mathbf{S}_{2}$ represents a coordinate system fixed relative to the moving fetal brain. Different processing stages operate either in $\mathbf{S}_{1}$ or $\mathbf{S}_{2}$, and are schematically represented in Figure 3 (all reported symbols will be defined later on).

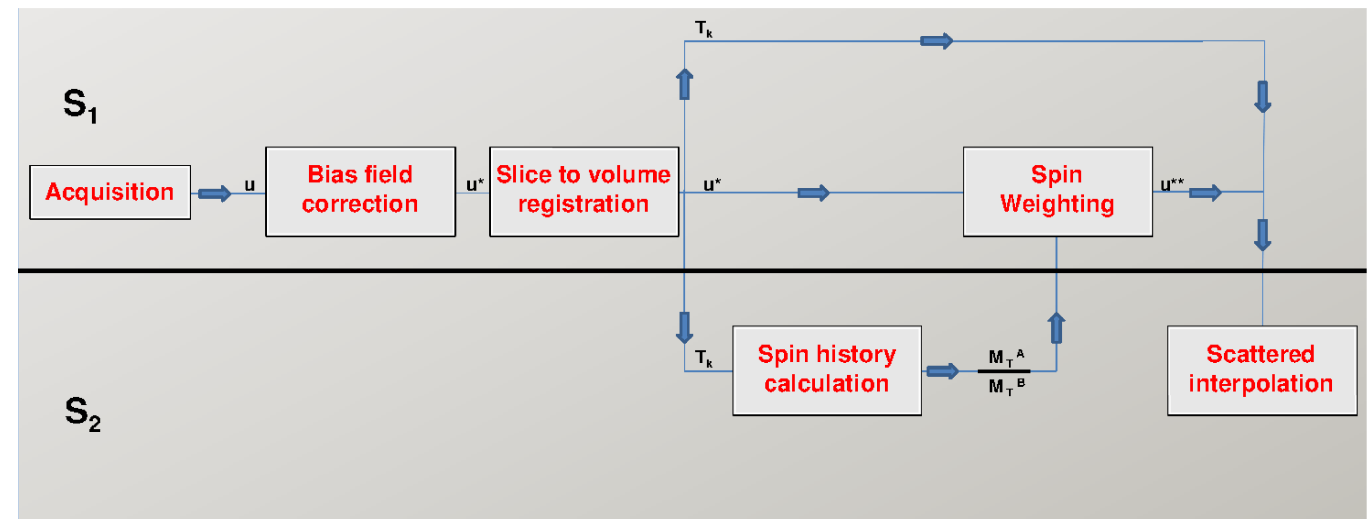

Figure 3: Processing Pipeline.

The proposed framework is divided into 4 different parts: prior to any motion correction, bias field correction is applied to remove spatially variable receiver coil sensitivity. This is done in the native scanner coordinates since the bias field is fixed relative to the maternal anatomy. Following this, a robust slice to volume registration realigns each acquired slice into a consistent anatomical space. Information from image registration is then incorporated into a forward model of the imaging process to quantify saturation effects so that fMRI data can be corrected for these incidental fluctuations. Finally, the corrected scattered data in $\mathbf{S}_{2}$ space is interpolated onto an uniform voxel grid so that it is suitable for subsequent fMRI analysis. Two different interpolation approaches for this important step were tested using simulated data for which a ground truth is known. The method with best performances was selected and used on in vivo data.

\subsubsection{Bias Field Correction}

As a preprocessing step, a binary mask was manually delineated around each fetal head. Masks were kept as close as possible to the fetal brain, but needed to be large enough to contain its full range of motion. 
Inhomogeneity of the radio-frequency receive sensitivity $\left(B_{1}\right)$ effects the voxel intensities of the acquired fMRI data as a multiplicative shading artefact (Juntu et al., 2005). The receiver coils are strapped to the mother's abdomen, and therefore $B_{1}$ is consistent in the space of acquisition $\left(\mathbf{S}_{1}\right)$ and does not vary with time provided the mother does not perform any gross movements. The coil elements are necessarily some distance from the fetal brain, so $B_{1}$ is generally slowly varying in space on the scale of the fetal head. However, due to fetal motion, this intensity variation is not consistent with respect to the fetal anatomy and so can effect both the performance of the registration algorithm used to perform motion-correction as well as the estimated fMRI signal.

There have been numerous studies in the literature attempting to perform correction of $B_{1}$ field inhomogeneity in MRI (Sled et al., 1998; Styner et al., 2000; Vovk et al., 2007). The majority of these methods dealt with high resolution $T_{1}$ or $T_{2}$ weighted images in adults and, in one case, anatomical $T_{2}$ weighted images in the fetus (Kim et al., 2011).

Unlike previous fetal MRI bias-correction methods (Kim et al., 2011; KuklisovaMurgasova et al., 2012) which were designed to correct bias field inconsistencies between acquired slices, our proposed method estimates the global volumetric bias field in the scanner space and can be considered an extension of the method proposed in the work of Wells III et al. (1996). We take advantage of the fact that our fetal fMRI data have very low contrast between different brain tissues (white matter, grey matter and cerebro-spinal fluid) and amniotic fluids. Only narrow regions of low signal are detected from bone in the skull.

To estimate $B_{1}$, we separate the voxels in the region of interest into two classes by fitting a mixture of Gaussians using the EM algorithm (Dempster et al., 1977; Duda et al., 2001). The multiplicative bias field impacts the higher intensity class much more significantly than the lower intensity class, thus separation of these two classes is preserved even in presence of relatively high $B_{1}$ inhomogeneity and we found it effective for all our datasets.

Voxels classified as having high intensities are used to fit a model for the bias field. Due to the low resolution, the data are strongly effected by partial volume effects that can influence the estimation of the bias field. Partial volume voxels are on the boundary between the two classes, and are therefore removed from the estimation using an erosion operation on the high-intensity class.

Let $\mathbf{x}_{i}$ denote locations of acquired voxels and $u_{j}\left(\mathbf{x}_{i}\right)$ their intensities in the $j^{\text {th }}$ 
frame. Let $s_{i j}$ be a segmentation of the high-intensity class, such that it is one if the voxels belong to this class and zero otherwise. Let $u_{j}^{*}\left(\mathbf{x}_{i}\right)=u_{j}\left(\mathbf{x}_{i}\right) e^{-b\left(\mathbf{x}_{i}\right)}$ denote the bias corrected intensities (in this formulation the bias field is modelled as $B_{1}\left(\mathbf{x}_{i}\right)=e^{b\left(\mathbf{x}_{i}\right)}$ and therefore its estimation is equivalent to the estimation of $b$ ).

Similarly to work presented by Wells III et al. (1996) and Kuklisova-Murgasova et al. (2013), we estimate the bias field by fitting a non-parametric regularized field. Specifically, the estimation of $b$ is performed by minimizing the following cost function:

$$
b=\underset{b}{\operatorname{argmin}} \sum_{i j} s_{i j}\left(u_{j}\left(\mathbf{x}_{i}\right) e^{-b\left(\mathbf{x}_{i}\right)}-\mu\right)^{2}+R(b)
$$

where $\mu$ is the mean intensity of the fMRI voxels and $R(b)$ a regularisation term that ensures spatial smoothness of $b$.

To minimize 1 an iterative strategy is adopted; the estimation of $b$ at a generic iteration $n$ is interleaved with the calculation of the mean intensity

$$
\mu^{n}=\frac{\sum_{i j} s_{i j}\left(u_{j}^{*}\left(\mathbf{x}_{i}\right)\right)^{n}}{\sum_{i j} s_{i j}}
$$

which serves as the estimated mean of the bias free intensities. $b$ can therefore be estimated by comparing the corrected acquired intensities to this value. At each iteration we calculate the bias residuals $r_{j}^{n}\left(\mathbf{x}_{i}\right)=\log \left(\left(u_{j}^{*}\left(\mathbf{x}_{i}\right)\right)^{n} / \mu^{n}\right)$. This is followed by weighted spatial (3D) Gaussian smoothing of the residuals $r_{j}^{n}\left(\mathbf{x}_{i}\right)$ in each time-frame with weights $w_{i j}^{n}=\left(u_{j}^{*}\left(\mathbf{x}_{i}\right)\right)^{n} s_{i j}$ and averaging in time to calculate the residual $\triangle b^{n}\left(\mathbf{x}_{i}\right)$ still present at the $n^{\text {th }}$ iteration

$$
\triangle b^{n}\left(\mathbf{x}_{i}\right)=\frac{1}{N_{F}} \sum_{j} \frac{\sum_{l} w_{l j}^{n} \phi_{\sigma_{b}}\left(d_{i l}\right) r_{j}^{n}\left(\mathbf{x}_{l}\right)}{\sum_{l} w_{l j}^{n} \phi_{\sigma_{b}}\left(d_{i l}\right)}
$$

where $d_{i l}$ represents the euclidean distance between voxels $i$ and $l, \phi_{\sigma_{b}}$ is a Gaussian kernel with standard deviation $\sigma_{b}$ for the smoothness of $b$, and $N_{F}$ is the number of time-frames. The bias-corrected image is then updated as follows: $\left(u_{j}^{*}\left(\mathbf{x}_{i}\right)\right)^{n+1}=\left(u_{j}^{*}\left(\mathbf{x}_{i}\right)\right)^{n} e^{-\triangle b^{n}\left(\mathbf{x}_{i}\right)}$.

\subsubsection{Slice to Volume Registration}

Echo Planar Imaging (EPI) in single shot mode allows the acquisition of a single slice in about $100 \mathrm{~ms}$, and so intra-slice motion artefacts are generally 
small in the fetal brain and we will not model these in what follows. Interslice motion is, however, still present and can be many millimeters or even many centimeters in scale. In this work we have so far not accounted for image distortions and so have applied a rigid body motion model for the correction of each slice.

Motion correction methods seek a geometrical transformation of the data to place it in a self consistent coordinate system in which the fetal brain appears to be stationary (Rousseau et al., 2006; Jiang et al., 2007; KuklisovaMurgasova et al., 2012). Here we used the approach developed by Jiang et al. (2007), in which a hierarchical registration pipeline was built and successfully applied to reconstruct anatomical $T_{2}$ weighed fetal brains with high SNR levels.

The processing pipeline is organized as follows (see Figure 4). As a first step, complete stacks of slices (fMRI time series plus coronal and sagittal volumes) are registered and a reference image is formed by averaging the entire dataset. Data are then divided into temporally contiguous blocks (in Figure 4 each block is color coded), containing fewer slices. Rigid body registration to the reference is then performed, and the reference image is subsequently updated. This process is repeated, with progressively smaller temporal groupings, stopping when each block contains one slice. The last iteration of the algorithm corresponds to direct slice to volume registration. The cost function minimized by the algorithm is sum of squared difference (SSD).

This process results in a set of rigid transformations that map all the slices from the fixed (i.e. stationary) scanner coordinate system $\mathbf{S}_{1}$ into the moving fetal brain reference system $\mathbf{S}_{2}$. Using a single cumulative strictly positive index $k$ to label each slice acquired during the whole fMRI time series we write:

$$
\mathbf{S}_{2, k}=\mathbf{T}_{k} \mathbf{S}_{1}
$$

where $\mathbf{T}_{k}$ is a 6 degrees of freedom transformation applied to the $k^{\text {th }}$ slice which was acquired at absolute time $(k-1) T_{S}$. In this formulation the first slice is assumed to be acquired at time zero.

\subsubsection{Correction of Spin History Artefacts}

Spin history artefacts will appear as a natural consequence of motion, where successive slices acquired at the same scanner coordinate system are effectively sampling the fetal brain at different anatomical positions. Changes of 


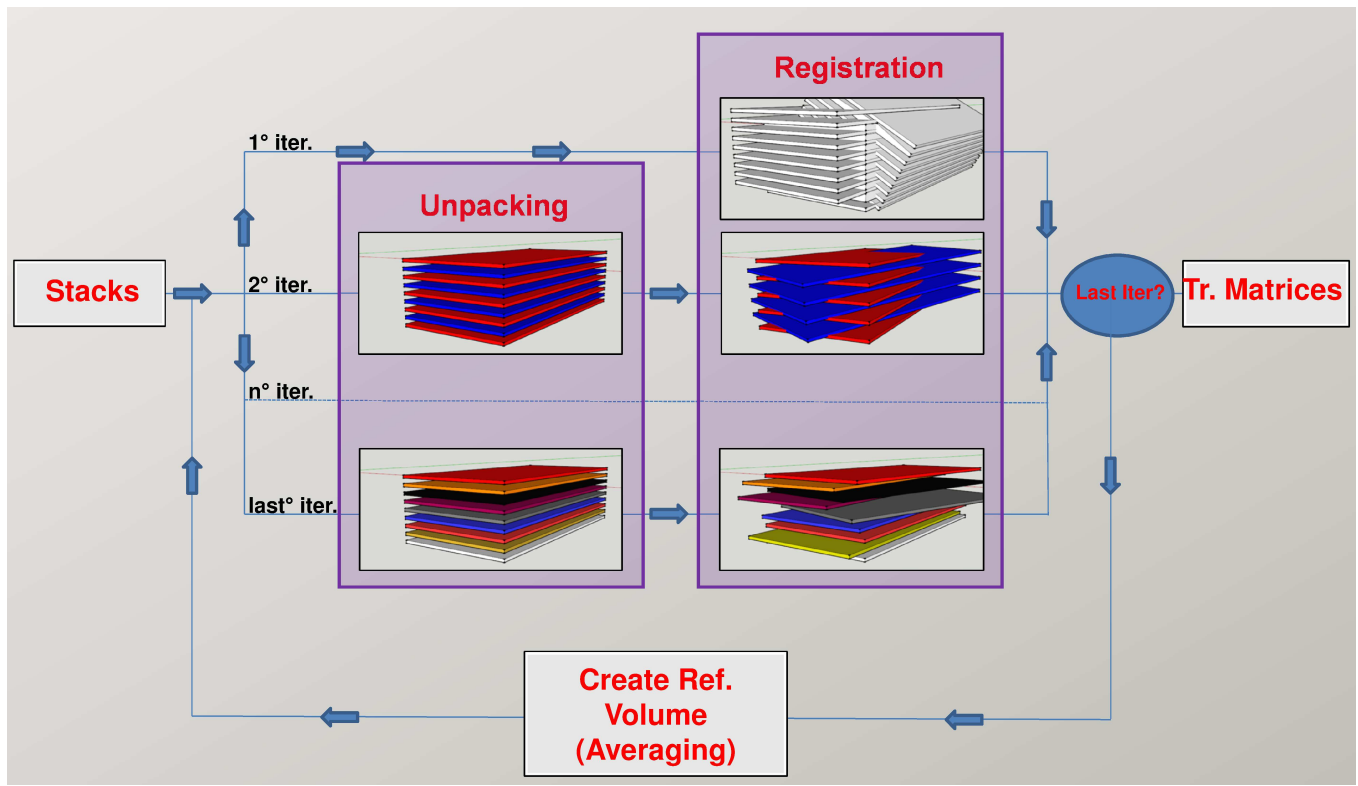

Figure 4: Motion correction Pipeline. Here each temporal block is color-coded and it represents the temporal unpacking of the data acquired during an interleaved acquisition.

the baby's position will therefore disrupt the steady state of the longitudinal magnetization ( $T_{2}$ decay is assumed to remove any significant coherence effects between successive excitations), and each measure is then effected by an unknown scaling factor (Bhagalia and Kim, 2008). To model spin history effects, we incorporate information from image registration into a forward model of the longitudinal and transverse magnetization components $\left(M_{L}^{k}\right.$ and $M_{T}^{k}$ ) immediately after the $k^{\text {th }}$ slice has been excited.

If no motion occurs $\left(\mathbf{S}_{1}=\mathbf{S}_{2}\right)$, the evolution of these two quantities at the time scale of $T_{S}$ is given by (Haacke et al., 1999):

$$
\left\{\begin{array}{l}
M_{L}^{k}(\mathbf{x})=\left[M_{0}-\left(M_{0}-M_{L}^{k-1}(\mathbf{x})\right) \cdot e^{-\frac{T_{S}}{T_{1}}}\right] \cos \left[\alpha \cdot \phi\left(\left|\mathbf{x}-\mathbf{x}_{\text {slice }_{k}}\right|\right)\right] \\
M_{T}^{k}(\mathbf{x})=\left[M_{0}-\left(M_{0}-M_{L}^{k-1}(\mathbf{x})\right) \cdot e^{-\frac{T_{S}}{T_{1}}}\right] \sin \left[\alpha \cdot \phi\left(\left|\mathbf{x}-\mathbf{x}_{\text {slice }_{k}}\right|\right)\right]
\end{array}\right.
$$

where $\mathbf{x}$ a generic position in $\mathbf{S}_{1}$ and $\mathbf{x}_{\text {slice }_{k}}$ its perpendicular projection onto the centerline of slice $k . M_{0}=1$ is the normalized longitudinal magnetization at equilibrium, $T_{1}$ the longitudinal relaxation time, $\alpha=90^{\circ}$ is the nominal flip 
angle used at acquisition and $\phi$ the slice excitation profile. In this work, $\phi$ is approximated as a Gaussian function of FWHM of $5 \mathrm{~mm}$ such that $\phi(0)=1$, and $T_{1}$ set at a typical value for neonatal brain of $1500 \mathrm{~ms}$ (Williams et al., 2005). The model steps through time units of $T_{S}$, updating the current state of magnetization $\left(M_{L}^{k}(\mathbf{x})\right.$ and $\left.M_{T}^{k}(\mathbf{x})\right)$ using the longitudinal magnetization computed at the previous iteration $\left(M_{L}^{k-1}(\mathbf{x})\right)$. As initial conditions, the longitudinal and transverse magnetization components $M_{L}^{0}(\mathbf{x})$ and $M_{T}^{0}(\mathbf{x})$ were set to $M_{0}$ and 0 respectively.

Assuming that slice to volume registration provides the correct transformation matrices to map slices from $\mathbf{S}_{1}$ into $\mathbf{S}_{2}$, the equations for the longitudinal and transverse magnetization components in the presence of motion become:

$$
\left\{\begin{array}{l}
M_{L}^{k}\left(\mathbf{y}_{k}\right)=\left[M_{0}-\left(M_{0}-M_{L}^{k-1}\left(\mathbf{y}_{k-1}\right)\right) \cdot e^{-\frac{T_{S}}{T_{1}}}\right] \cos \left[\alpha \cdot \phi\left(\left|\mathbf{y}_{k}-\mathbf{y}_{\text {slice }_{k}}\right|\right)\right] \\
M_{T}^{k}\left(\mathbf{y}_{k}\right)=\left[M_{0}-\left(M_{0}-M_{L}^{k-1}\left(\mathbf{y}_{k-1}\right)\right) \cdot e^{-\frac{T_{S}}{T_{1}}}\right] \sin \left[\alpha \cdot \phi\left(\left|\mathbf{y}_{k}-\mathbf{y}_{\text {slice }_{k}}\right|\right)\right]
\end{array}\right.
$$

where $\mathbf{y}_{k}=\mathbf{T}_{k} \mathbf{x}$ and $\mathbf{y}_{\text {slice }_{k}}=\mathbf{T}_{k} \mathbf{x}_{\text {slice }_{k}}$.

We run both equations 5 and 6 on a $1 \mathrm{~mm}$ isotropic grid, obtaining a high resolution estimate of the magnetization components for each subject for each slice both with and without the estimates of motion.

Since spin history corrections are applied directly to the original moving data (defined in $\mathbf{S}_{1}$ at the original coarse resolution), in the case of no motion, all the samples within each voxel $\mathbf{x}_{i}$ in $\mathbf{S}_{1}$ of sizes $2.5 \cdot 2.5 \cdot 5 \mathrm{~mm}^{3}$ (in-plane resolution versus slice thickness) were averaged. For equation 6 , all voxels at the fine resolution scale that were falling into $\mathbf{y}_{i, k}$ (projection of $\mathbf{x}_{i}$ into $\mathbf{S}_{2, k}$ ) were also averaged, and stored the resulting value at the original location in $\mathrm{S}_{1}$.

This produced two estimates of the transverse magnetizations, labelled respectively $M_{T}^{j, A}\left(\mathbf{x}_{i}\right)$ and $M_{T}^{j, B}\left(\mathbf{x}_{i}\right)$, defined for each frame $j$, at the original coarse resolution relative to the scanner coordinate system $\mathbf{S}_{1}$. The labels $B$ and $A$ stand for the transverse magnetization components that are obtained when the presence of motion is and is not simulated.

To remove the variable saturation effects caused by intra-slice motion, each bias corrected fMRI voxel $u_{j}^{*}\left(\mathbf{x}_{i}\right)$ was weighted using the following formula:

$$
u_{j}^{* *}\left(\mathbf{x}_{i}\right)=u_{j}^{*}\left(\mathbf{x}_{i}\right) \frac{M_{T}^{j, A}\left(\mathbf{x}_{i}\right)}{M_{T}^{j, B}\left(\mathbf{x}_{i}\right)}
$$


with $u_{j}^{* *}\left(\mathbf{x}_{i}\right)$ representing the bias-spin corrected voxel acquired within the $j^{\text {th }}$ frame at location $\mathbf{x}_{i}$.

\subsubsection{Scattered Data Interpolation}

The transformations determined by image registration provide a direct means of transforming from the original slice positions in $\mathbf{S}_{1}$ to the corresponding positions in $\mathbf{S}_{2}$. The slice data are irregularly positioned in $\mathbf{S}_{2}$ and need to be interpolated onto a uniform grid for further fMRI analysis. This is a key part of the pipeline where data can be degraded so we tested two different scattered data interpolation strategies, utilising both spatial and temporal proximity in different ways. Each method performs locally linear interpolation based on Delaunay triangulation (Delaunay, 1934).

Although complete stacks of fMRI slices are repeatedly acquired, each individual slice is a single-shot, which results in systematic timing offsets for each slice in scanner coordinates (in steps of $T_{S}$ ). Also, temporally adjacent slices do not necessarily have to be spatially adjacent, as slice time ordering can be chosen by the operator. There are thus two clear options; to regard the data as scattered in a 3D space, grouped into coarse time bins of duration $T_{R}$ (stack repeat time), or to treat all the data as $4 \mathrm{D}$ space-time observations in which the distance between locations depends on both the spatial and temporal separation. To create a distance metric in space-time, we scaled the time dimension by a factor of $\frac{\operatorname{res}(z)}{T_{R}}$, where $\operatorname{res}(z)$ is the resolution of the image in the through slice direction.

Delaunay Linear interpolation: the Delaunay triangulation constructs a mesh by connecting nearest neighbours with straight edges. In $2 \mathrm{D}$ this results in a mesh of triangles, in 3D a mesh of tetrahedrons and so on. A Delaunay mesh is a tessellation such that the circumspheres associated with each simplex do not contain any other vertices in their interior. As a consequence of that, the Delaunay tessellation ensures that vertices belonging to the same simplex are also nearest neighbours. This property of the Delaunay mesh renders it particularly suitable for efficient scattered data interpolation, providing a means to estimate a value at a specific location from values in the local neighbourhood (De Berg et al., 2008). In this case we used piece-wise linear interpolation from the vertices for each simplex that contains the target point.

The choice of Delaunay interpolation over previously proposed multilevel Bsplines scattered data interpolation (Jiang et al., 2007) and super-resolution 
reconstruction approaches (Kuklisova-Murgasova et al., 2012) is due to the following reason; while reconstruction of anatomical $T_{2}$ weighted images can exploit an oversampling factor that comes naturally by imaging the fetal brain multiple times for multiple views, in the context of fetal fMRI what is really desired is the reconstruction of time series. The consequence of this is that the natural rate of scattered positions versus points to infer is 1 to 1 . This relationship may also get worse when big motion occurs, by opening up holes between slices that are spatially adjacent.

B-splines can be unreliable in this type of application because of isotropic kernels and an inability to fill areas with missing data. Furthermore super resolution approaches are suitable when oversampling in the data can be used to improve its resolution. In fetal fMRI data there is no oversampling and therefore their usage is very undesirable.

\subsubsection{Validation of Interpolation Methods}

A forward simulation was used to validate the interpolation schemes. In the first step, a synthetic dataset was constructed by taking a fetal head mask and defining a set of five spatial regions, color coded in Figure 5. Each mask was spatially smoothed to avoid ringing effects when resampling. A specific synthetic time course was assigned to each of these regions as follows: $s_{1}(t)=\sin (2 \pi \cdot 0.05 \cdot t), s_{2}(t)=\cos (2 \pi \cdot 0.01 \cdot t), s_{3}(t)=\operatorname{sawtooth}(2 \pi \cdot 0.02 \cdot t)$, $s_{4}(t)=\operatorname{repbox}(2 \pi \cdot 0.03 \cdot t), s_{5}(t)=\sin (2 \pi \cdot 0.08 \cdot t)$, with sawtooth and repbox representing a train of repeated sawtooth and boxcar functions respectively. A global mean of 500 units was assigned to the entire volume mask.

The simulation was set up at the time scale of $T_{S}$. To match the temporal structure of real data acquisition, a $T_{R}=4 \mathrm{sec}$ and a total number of 35 slices for each stack were used. The spatial resolution of the simulated data was $1 \mathrm{~mm}$ isotropic.

The first step was to model the sampling of the object that the scanner performs. Initially, the transformations between $\mathbf{S}_{1}$ and $\mathbf{S}_{2}$ were set to identity, so that the object was stationary in $\mathbf{S}_{1}$. The simulated slice data consisted of voxels $\mathbf{x}_{i}$ in $\mathbf{S}_{1}$ of size of $2.5 \cdot 2.5 \cdot 5 \mathrm{~mm}^{3}$ (in-plane resolution versus slice thickness). The simulated "acquired" signals were calculated as the mean of the values from all the voxels in the high-resolution model that fall within $\mathbf{x}_{i}$. In order to investigate possible effects of different slice overlaps on the final result, complete simulations were run with overlaps of $1 \mathrm{~mm}$ and 2.5 $\mathrm{mm}$, which correspond to the minimum and maximum amount of overlap in the real data. Acquisition slice ordering was interleaved (1-3-5-7...2-4-6-8...) 


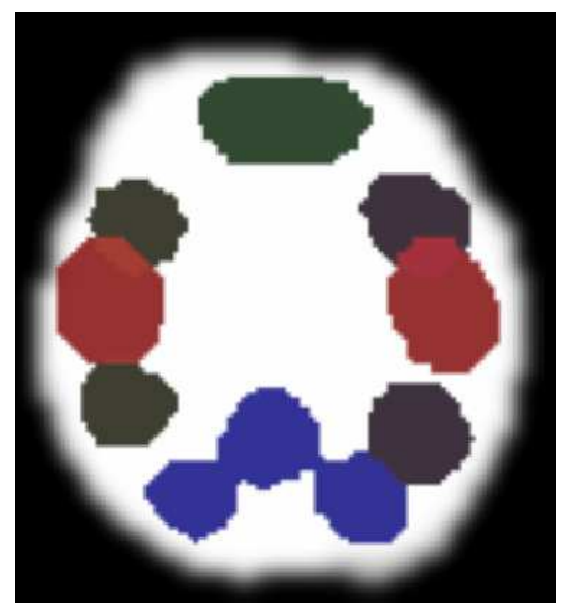

Figure 5: Spatial distribution of the five regions used in the forward simulation.

and 100 volumes were acquired.

After simulated acquisition, i.i.d. Gaussian noise was added to each voxel. The variance of the noise was selected to provide a low BOLD effect-tonoise ratio, typical of RSNs, where the explained variance in adult studies is around 2 or $3 \%$. The simulated data were then analyzed using Probabilistic ICA as implemented in FSL Melodic (http://fsl.fmrib.ox.ac.uk/fsl/fslwiki/) to determine if the temporal-spatial patterns built into the model could be detected.

ICA is a data driven method used to find a set of components in which statistical independence is maximized (Hyvärinen and Oja, 2000). In the context of fMRI, ICA splits the data into a set of spatial statistically independent components, each of which is associated with an unique time trend. Probabilistic ICA includes isotropic noise in the definition of the model to allow for the uncertainties associated with each measure.

Before running Probabilistic ICA, data were spatially smoothed with a Gaussian Kernel of FWHM of $2 \mathrm{~mm}$.

Motion was then simulated by applying to each slice in $\mathbf{S}_{1}$ the set of rigid transformations observed in the subject in our study population that exhibited the largest level of movement. Each "acquired" voxel, $\mathbf{x}_{i}$, in $\mathbf{S}_{1}$ was therefore projected into $\mathbf{S}_{2, k}$, obtaining its projected version $\mathbf{y}_{i, k}$. The signal for each position in $\mathbf{S}_{1}$ was then assigned to the average of the voxel values at the fine resolution that were within $\mathbf{y}_{i, k}$. Independent Gaussian noise with 
the same variance as before was then added to the signal for each acquired voxel in the moving object in $\mathbf{S}_{1}$.

Finally, to test each interpolation scheme, the simulated acquired (noisy) slice data in $\mathbf{S}_{1}$ was projected back into $\mathbf{S}_{2}$ to produce scattered data ready for analysis. Regular spaced data was then recovered using each of the interpolation schemes outlined in section 2.2.4 and "Resting State Networks" identified using standard Probabilistic ICA, after smoothing with a Gaussian Kernel of $2 \mathrm{~mm}$.

\subsubsection{Group Independent Component Analysis}

Group Probabilistic ICA was used to discover Resting State Networks at the population level from the fetal data after correcting for bias field, spin history and motion. Brain extraction was performed on each subject using a manually delineated fetal brain mask.

The data from all subjects were placed into a common space defined by selecting a fetal $T_{2}$ weighted atlas of 32 weeks of gestation (the mean gestational age of the group). The atlas was created from separate data according to the method described by Serag et al. (2012). The required registrations were carried out in two separate steps; first, for each subject separately, the fMRI data were rigidly registered to their corresponding $T_{2}$ weighted brain volume produced from the ssFSE images that had been realigned and reconstructed as described in Jiang et al. (2007). The $T_{2}$ weighted volume reconstructions were aligned to the common target space using affine registration. The individual reconstructed fetal brain volumes achieve self-consistency, but may appear in an arbitrary pose. It was therefore frequently necessary to supply a starting transformation estimate. The transformations obtained by the two registration processes were composed and applied to fetal fMRI data in a single step. Finally, each dataset was spatially smoothed with a Gaussian Kernel of $2 \mathrm{~mm}$.

\section{Results}

The following sections first report the results obtained on the synthetic dataset, and then those obtained from in vivo data.

\subsection{Scattered Data Interpolation}

Figure 6 shows the components recovered by Probabilistic ICA for the $1 \mathrm{~mm}$ and $2.5 \mathrm{~mm}$ overlap cases, when no motion is included in the simulation 
$\left(\mathbf{S}_{1}=\mathbf{S}_{2}\right)$. The Figure shows z-score maps thresholded using the cut-off value given by FSL Melodic. When running ICA the number of components was automatically estimated correctly to be five. As expected, in both cases, ICA is capable of recovering spatial-temporal patterns, that, if compared with Figure 5, demonstrate a good level of overlap.

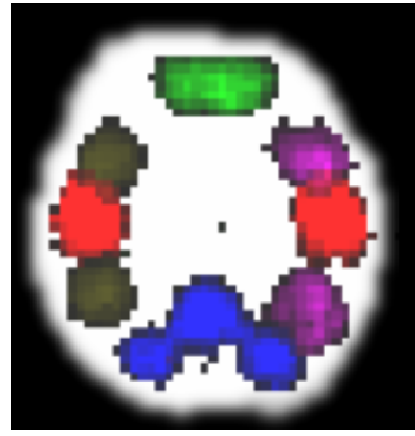

(a) Overlap of $1 \mathrm{~mm}$.

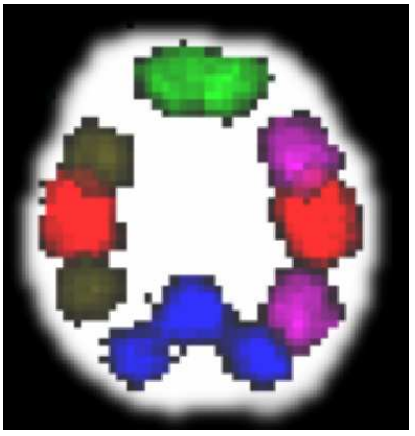

(b) Overlap of $2.5 \mathrm{~mm}$.

Figure 6: Components recovered by ICA for the two different levels of overlap.

Running ICA on the moving object without motion correction does still partially recover some signal for the 5 components, but with substantial damage (Figure 7).

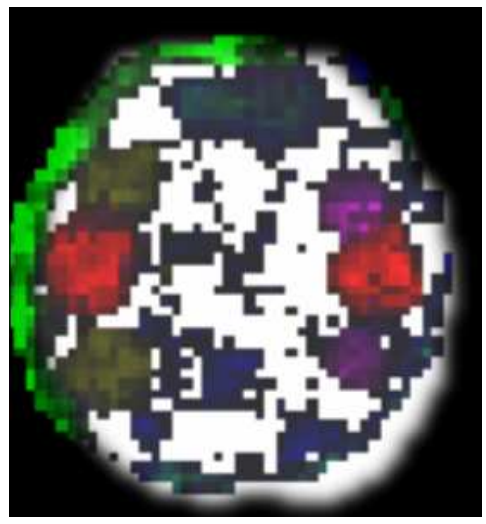

Figure 7: Spatial distribution of the 5 components in a moving simulated dataset when motion is not corrected. Here we are showing the result with slice overlap of $1 \mathrm{~mm}$. 
Figure 8 shows the recovered components obtained on the motion corrected objects after having performed scattered interpolation. Columns show the result obtained by using Delaunay $3 \mathrm{D}$ and 4D, and rows recovered maps for the two different level of overlap. In all cases, we are able to recover the introduced components, with no substantial differences between the two levels of overlap.

To evaluate the performances of the two different scattered interpolation schemes, we calculated the normalized root mean square error (NRMSE) between the motion free dataset (obtained when $\mathbf{S}_{1}=\mathbf{S}_{2}$ ) and motion corrected ones. The calculation was carried out on all noise free datasets and the NRMSE (in \%) is shown in Figure 9.

Based on a visual inspection and the NRMSE results, the Delaunay 3D method appears to perform best for the problem in question, and we subsequently decided to apply this method to in vivo data.

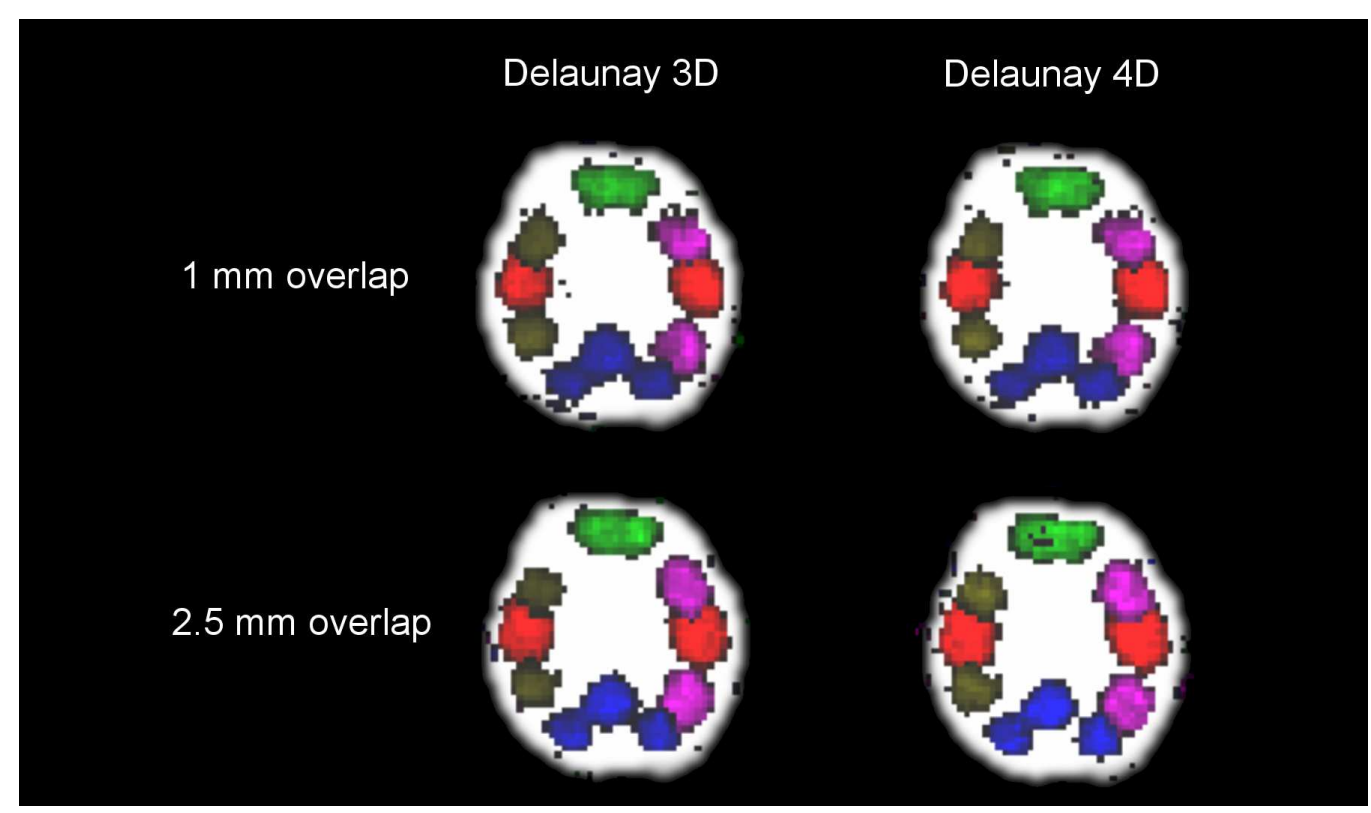

Figure 8: Spatial distribution of the 5 components for Delaunay 3D and 4D and for the two different overlaps each of which is corrected for motion. 

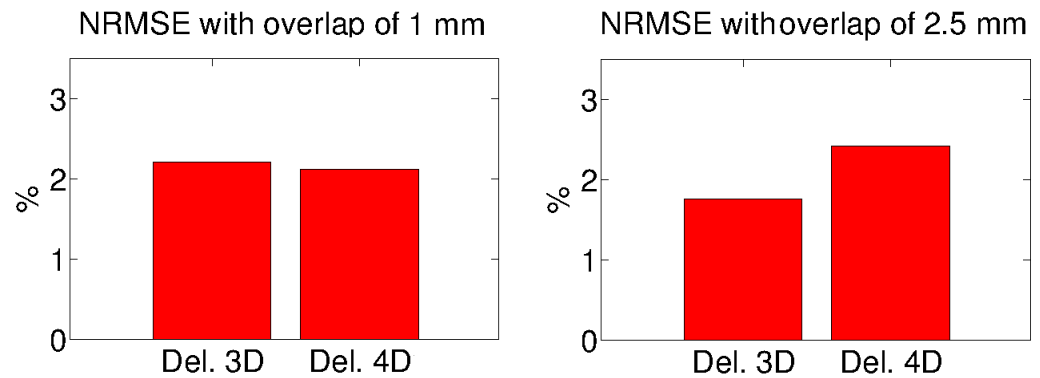

Figure 9: NRMSE for all scattered interpolation methods and for the two levels of overlap.

\subsection{Bias Field Correction}

Figure 10 shows a slice in the transverse plane before (a) and after (b) bias correction for one of the subjects that presented with strong bias effects. The shading given by the inhomogeneities of $B_{1}$ is largely resolved. The estimated bias field is shown in Figure 10c; its application results in much more homogeneous image properties, although this is hard to quantify in the absence of a ground truth estimate.

Means and standard deviations for the estimated bias fields over the region of interest were $1.024 \pm 0.138,1.036 \pm 0.223,0.994 \pm 0.106,1.005 \pm 0.120$, $0.932 \pm 0.154,1.002 \pm 0.069,0.993 \pm 0.049$ and $1.007 \pm 0.078$.

We have finally performed an exhaustive simulation on the data from this study to measure the performances of our bias correction algorithm. Having corrected every subject, we applied all the resulting bias field estimates to the corrected images of every other subject. Each bias field was projected and interpolated onto the space defined by the fetal head and the bias field applied to each image independently. We therefore obtained a set of 56 examples, each of which was characterized by a different subject-bias field combination.

The bias correction algorithm was then run on this set of 56 images, and the performances measured by calculating the NRMSE between the estimated bias fields and the corresponding ground truth. This led to the Table reported in Figure 11, where each row represents a subject, and each column the associated bias field. The NRMSE was 1-2\% for the majority of the cases, reaching peaks of 5-6\% when using the bias field from subject number 5 (fifth column). Despite this bigger error, we observed good results even in this case; the bottom part of same Figure reports images before and after 


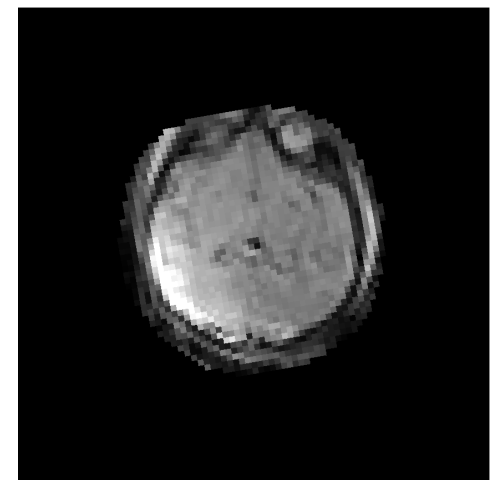

(a) Image presenting strong bias effect.

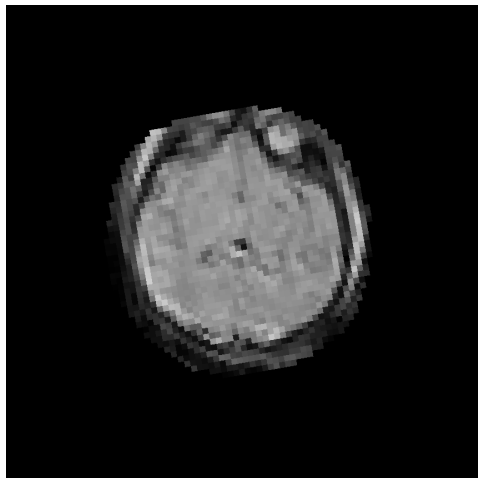

(b) Image after bias correction.

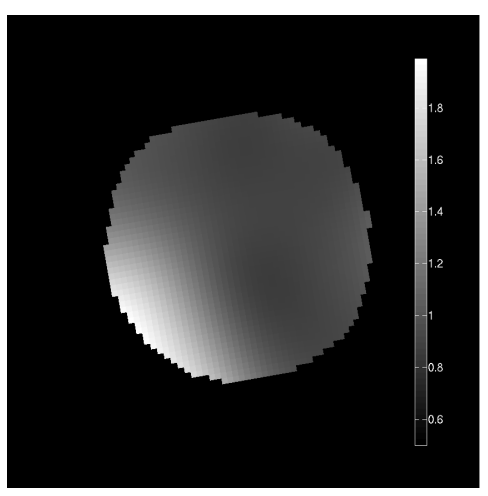

(c) Bias field.

Figure 10: Bias field correction results. An EPI slice (a) before and (b) after bias field correction using the bias field shown in (c).

bias field correction using this $B_{1}$ map, and shows a substantial reduction of inhomogeneities in all cases. 
a)

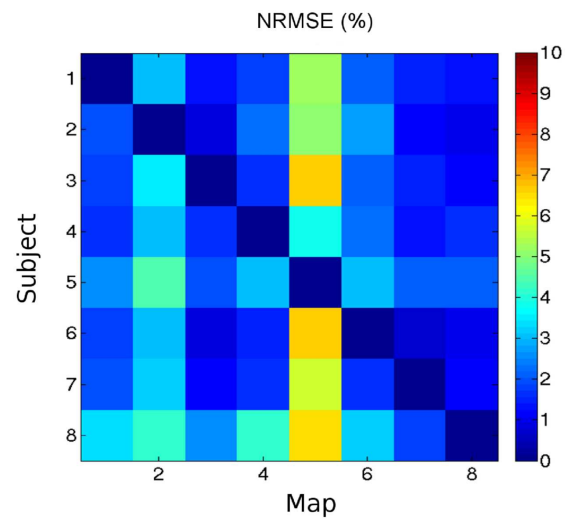

b)

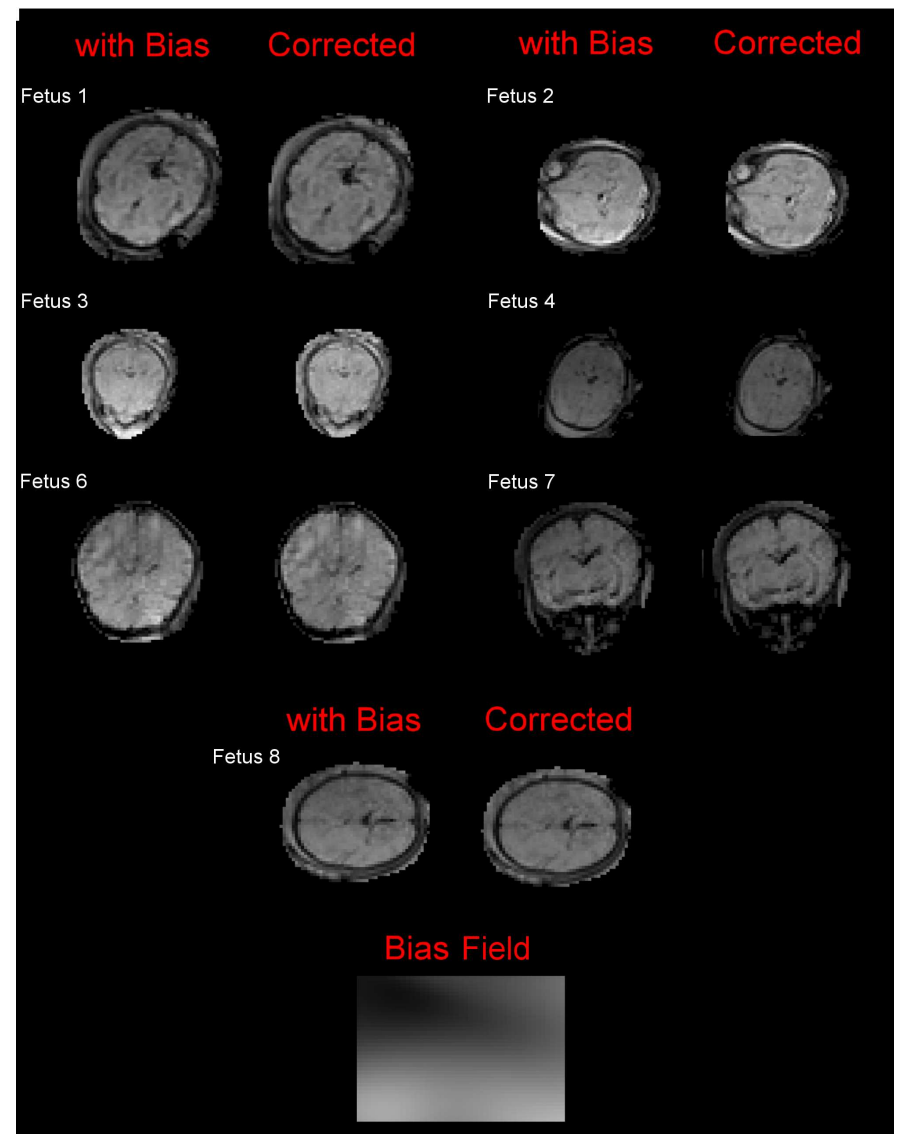

Figure 11: (a) NRMSE between estimated bias and ground truth in the permutation test. (b) Comparison between the 7 subjects corrupted with bias field number 5 and the corresponding bias corrected images. 


\subsection{Slice to Volume Registration}

In order to assess the registration performances, we used a forward simulation. We chose from our cohort of registered subjects the one that exhibited the highest level of motion (fetus number one from Table 1) and selected one volume with no substantial motion artefacts as reference. The entire volume was then projected into the space of acquisition by using the transformation matrices that had been previously computed when registering the original data. The signal coming from the projected volumes was then interpolated onto the space defined by each slice using B-splines (Lee et al., 1997). The use of B-Splines is here convenient as the scattered space formed by the projected volumes is regular. Isotropic noise was finally added to each voxel to reach typical levels of SNR.

The synthetic moving dataset was then fed into the registration framework and the estimated motion parameters compared with the ground truth. Means and standard deviations of the absolute error were $0.047 \pm 0.066 \mathrm{~mm}, 0.039$ $\pm 0.075 \mathrm{~mm}, 0.066 \pm 0.096 \mathrm{~mm}$ for translations, and $0.194 \pm 0.147^{\circ}, 0.174$ $\pm 0.130^{\circ}, 0.122 \pm 0.122^{\circ}$ for rotations.

Table 2 summarizes motion of all the corrected subjects showing that there was substantial translation and especially rotation. The grey row of the Table reports the means and standard deviations of the motion parameters obtained from one of the three subjects for which the registration algorithm failed, whereas the blue one refers to one of those subjects that exceeded the field of view.

In the case of excessive motion, the registration procedure reduced the observable motion substantially, but residual movement was still clearly seen and at some time points the interpolated volumes were obviously damaged. The motion estimates, although incorrect, do give an indication of the magnitude of the motion exhibited, which was higher than all the other subjects in most motion parameters.

Figure 12 shows the estimated motion parameters plotted against the acquired volume index for subject number one. Parameters are arranged according to the slice acquisition order. The translations are comparable in magnitude with the dimensions of each voxel. Estimated rotations take values within the range $-20^{\circ}$ and $+10^{\circ}$ around the $x$ and $y$ axes, reaching a peak of $-60^{\circ}$ for rotations around the $z$ axis. To illustrate the accuracy of the registration algorithm, we focus on the red segment of Figure 12, which corresponds to volumes 23 and 24 in the original data and coincides to the 


\begin{tabular}{|c|c|c|c|c|c|c|}
\hline & \multicolumn{3}{|c|}{ Translation } & \multicolumn{3}{c|}{ Rotation } \\
\hline Fetus & $\mathrm{tx}(\mathrm{mm})$ & $\mathrm{ty}(\mathrm{mm})$ & $\mathrm{tz}(\mathrm{mm})$ & $\mathrm{rx}\left(^{\circ}\right)$ & $\mathrm{ry}\left(^{\circ}\right)$ & $\mathrm{rz}\left(^{\circ}\right)$ \\
\hline 1 & $-0.37 \pm 0.55$ & $1.10 \pm 0.72$ & $-1.69 \pm 0.82$ & $0.56 \pm 3.72$ & $0.48 \pm 2.15$ & $-4.41 \pm 7.21$ \\
\hline 2 & $-0.41 \pm 1.09$ & $-1.03 \pm 1.07$ & $-0.48 \pm 0.52$ & $1.52 \pm 3.32$ & $-0.93 \pm 4.03$ & $2.09 \pm 2.64$ \\
\hline 3 & $0.69 \pm 0.61$ & $-1.37 \pm 1.10$ & $1.36 \pm 1.13$ & $2.13 \pm 1.9$ & $1.01 \pm 1.5$ & $0.75 \pm 1.30$ \\
\hline 4 & $0.98 \pm 0.62$ & $-2.86 \pm 1.30$ & $2.94 \pm 1.97$ & $4.18 \pm 3.4$ & $3.4 \pm 1.91$ & $-0.78 \pm 1.47$ \\
\hline 5 & $1.66 \pm 1.70$ & $0.16 \pm 0.64$ & $0.42 \pm 1.19$ & $-0.28 \pm 2.27$ & $2.94 \pm 5.98$ & $1.18 \pm 5.49$ \\
\hline 6 & $0.04 \pm 0.59$ & $0.69 \pm 1.33$ & $0.35 \pm 0.76$ & $0.87 \pm 2.13$ & $0.97 \pm 3.12$ & $-1.84 \pm 4$ \\
\hline 7 & $-0.25 \pm 0.89$ & $0.98 \pm 1.27$ & $0.96 \pm 0.96$ & $-0.04 \pm 3.46$ & $0.25 \pm 3.05$ & $-0.79 \pm 2.91$ \\
\hline 8 & $0.61 \pm 1.12$ & $-0.53 \pm 0.88$ & $0.59 \pm 0.89$ & $0.14 \pm 2.07$ & $-2.42 \pm 3.59$ & $1.37 \pm 3.16$ \\
\hline 9 & $6.70 \pm 4.0$ & $-1.12 \pm-1.79$ & $-2.03 \pm 2.57$ & $13.53 \pm 16.38$ & $7.85 \pm 9.28$ & $8.59 \pm 17.73$ \\
\hline 12 & $-0.79 \pm 2.86$ & $3.33 \pm 2.97$ & $0.043 \pm 2.49$ & $0.79 \pm 5.27$ & $-0.79 \pm 4.66$ & $-7.26 \pm 9.57$ \\
\hline
\end{tabular}

Table 2: Mean and standard deviation of rigid body motion parameters for all the corrected subjects (1-8), for one of the fetuses for which the registration algorithm failed (9) and for one that exceed the field of view

period in which fetus one moved the most.

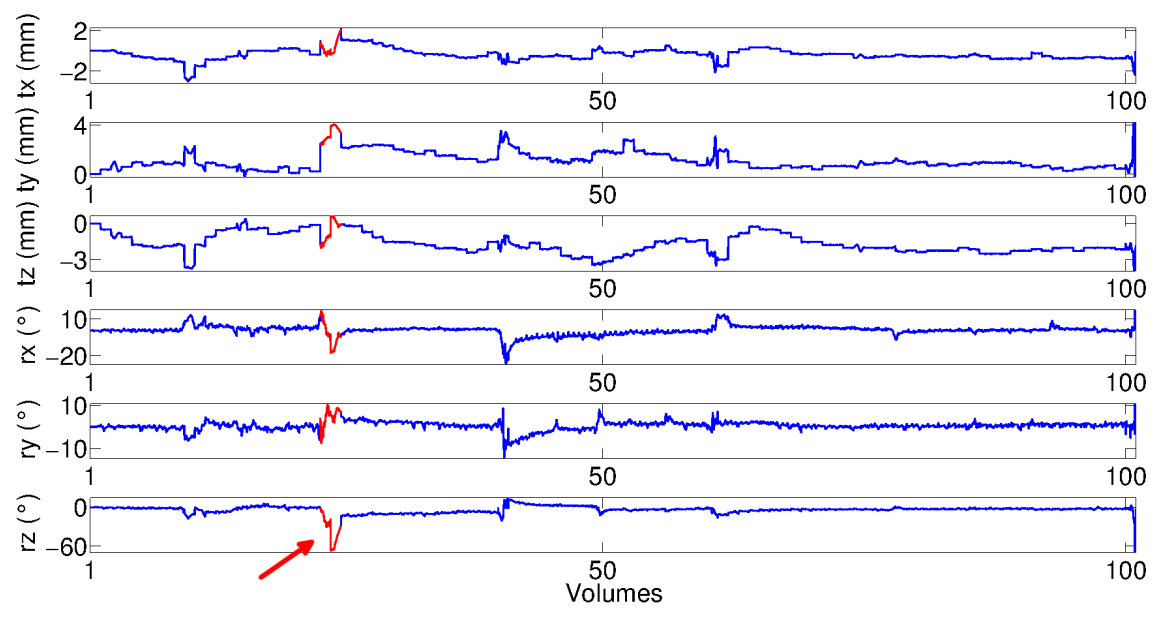

Figure 12: Behaviour of the estimated motion parameters. The red segments of the curves (indicated also by the red arrow) will serve as illustration of the performances of the registration later on.

Figure 13a shows an 8 second period of the time course. The same motion 
estimates, but rearranged in geometrical slice order, are shown in Figure 13b. The oscillating nature of the reconstructed motion parameters in b derives from the interleaved slice ordering used at acquisition. This oscillatory behaviour appears to decline at the edges of the stacks of slices in Figure 13b (region shown in lighter blue), where the acquired slices are in fact outside the brain at its superior and inferior extremes. These slices cannot be aligned to the target reconstructed brain during the final slice-by-slice stages of registration process and so their parameters remain static after the registration with other slices that are inside the brain; the hierarchical strategy proposed by Jiang et al. (2007) initializes the registration of each sub-block of slices with an initial transformation computed when performing the registration of the corresponding parent block. Sudden jumps of the motion estimates in Figure 13b between volumes 23 and 24 do not correspond to sudden movements, but are a natural effect of the registration framework.

Figure 13c (first two rows) shows slices taken at two different levels of the brain from volume stacks 23 and 24. The Figure provides a striking, but not untypical, illustration of how fetuses frequently change position, particularly by rotation within the time taken to acquire a single complete stack. The lower half of Figure 13c shows the same set of slice locations after motion correction. The corresponding motion estimates are also highlighted in Figure 13 with colored circles. 
a)
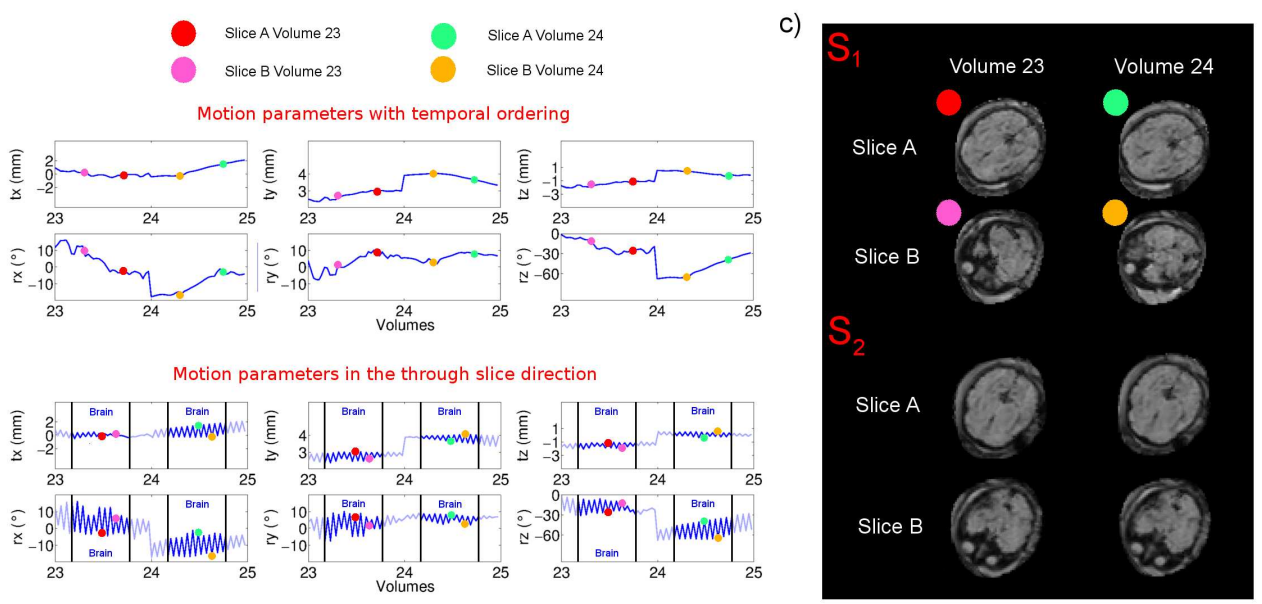

b)

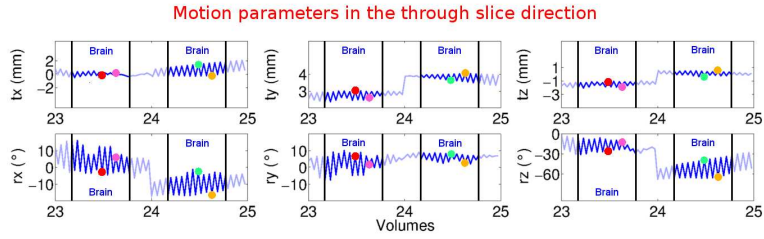

Figure 13: (a) Reconstructed motion parameters with temporal ordering for the red segment of Figure 12. (b) Motion parameters presented in geometrical slice order. Slices belonging to the brain are highlighted, those beyond the superior and inferior margins of the brain are in lighter blue.

(c) Two slices taken from the 3D slice stack at volumes 23 and 24 before motion correction, i.e. in scanner coordinates $\mathbf{S}_{1}$ (first two rows), and after motion correction, i.e. in patient coordinates $\mathbf{S}_{2}$ (last two rows).

Finally, in order to be able to provide a measure of absolute motion for those examples in which the registration algorithm failed, the pupil of the left eye was chosen as a landmark, and its motion tracked by taking the distance of its position between the 2 volumes of the time series in which the motion excursion was maximal. The same calculation was repeated for subjects labelled 12 to 14 of Table 1, but for these cases the start and end frames were the first volume of the time series and the volume in which the fetus first moved partly outside the imaged region. The calculated absolute displacements were $55.2,37.6$ and $36.2 \mathrm{~mm}$ for subjects 9,10 and 11 , and of 36.5 , 22.3 and $53.7 \mathrm{~mm}$ for subjects 12,13 and 14 .

\subsection{Correction of Spin History Artefacts}

The transverse magnetization at a specific space-time location in functional imaging is proportional to the stored longitudinal magnetization that is available at that spatial location just prior to the application of the excitation $\mathrm{RF}$ pulse. If the spin history model is correct then we would expect correlation between the time courses of the observed fMRI data and the predicted 
saturation factors. Conversely, if the model did not predict these effects, the saturation factors would not related to the data at all, so there would only be chance correlation.

We therefore calculated the cross correlation between the predicted time varying saturation factor $\left(M_{T}^{B}\right)$ at each location in anatomical space $\left(\mathbf{S}_{2}\right)$ and the recorded time series after motion correction. To do this, the saturation factors for each slice calculated in scanner coordinates $\mathbf{S}_{1}$ were transformed into $\mathbf{S}_{2}$ and interpolated onto a regular grid. Figures 14a shows the resulting correlation map for subject number one and suggests that the signal is generally correlated with the saturation time courses predicted by the model. Considering the subject group as a whole and taking the average correlation score across each brain shows mean values of $-0.79 \%$ for the least correlated subject and $24.63 \%$ for the most correlated one.

To test the performance of the model, a permutation test was conducted on subject number one. The saturation time courses for each voxel predicted by the model were randomly assigned to another voxel position in $\mathbf{S}_{2}$. Correlation maps between the original fMRI data and this new configuration of saturation factors was then recalculated. The procedure was repeated 1000 times and the mean correlation score calculated each time. We then tested the null hypothesis that these 1000 correlation scores are a realization of a Gaussian distribution. This null hypothesis was accepted $(p>0.05)$.

Finally, in order to see whether the original configuration (i.e. without reshuffling) of the saturation factors is capable of describing sensible variations in the data, we tested the hypothesis that its mean correlation score belongs to the Gaussian distribution computed in the previous step. This test had negative outcome as the mean correlation score was found to lie beyond 70 standard deviations from the center of the Gaussian distribution, confirming that the time saturation factors as given by the spin history model are correlated with the fMRI signals by more than chance and therefore justifying their removal.

To assess the sensitivity of the spin history model to changes of $T_{1}$, the estimation $M_{T}^{B}$ was repeated using a $T_{1}=3000 \mathrm{~ms}$ and the correlations with the fMRI data recalculated. Results for subject one are shown Figure 14c, and confirm that the correlation score does not depend strongly upon the choice of $T_{1}$. However, a t-test under the null hypothesis of the results in Figures $14 \mathrm{a}$ and $\mathrm{c}$ being realizations of Gaussian random variables with identical means and variances was rejected $(p<0.05)$, confirming that the choice of $T_{1}$ does influence the estimation of the saturation factor to be applied at 
each spatial-temporal voxel location in a detectable way.

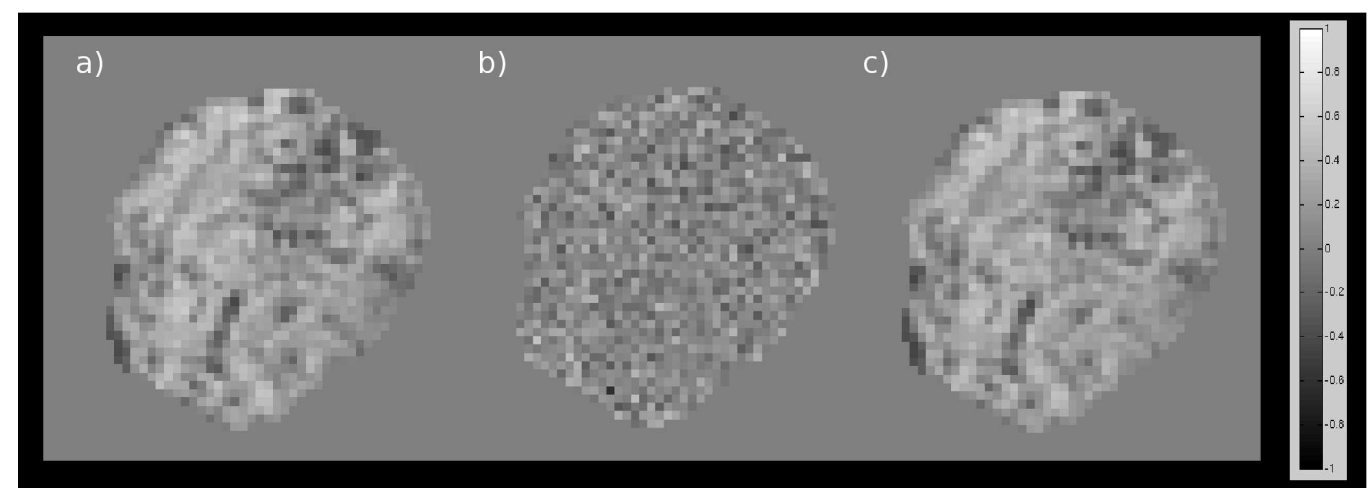

Figure 14: (a) Correlation score between $M_{T}^{B}$ given by the model and the corresponding fMRI time series $\left(T_{1}=1500 \mathrm{~ms}\right)$. (b) Correlation score between the same fMRI data with saturation factors from the permutation test. (c) Correlation score between original data and model prediction using

$$
T_{1}=3000 \mathrm{~ms} \text {. }
$$

\subsection{Group Independent Component Analysis}

Group ICA extracted 20 components, 6 of which (Figure 15) were identified as spatially resembling those previously described in preterm neonates by Smyser et al. (2010) and by Doria et al. (2010).

These consisted of: (A) lateral visual network encompassing the extra-striate visual areas; (B) a medial visual network comprising the striate and parastriate areas; (C) a bilateral sensori-motor network in the superior parietal lobe encompassing the primary motor and somatosensory cortices (anterior and posterior to the central sulcus respectively); (D) a primitive default mode network in the medial prefrontal and anterior cingulate cortices; (E) and (F) unilateral auditory networks in the superior temporal and insular areas in the left and right hemispheres respectively. In accordance with the findings of studies with preterm infants, a trend towards a more bilateral network representation (between one region and its homotopic counterpart) was seen in those encompassing medial areas (such as the sensori-motor and medial visual networks). In contrast, networks presumed to demonstrate more longrange connectivity in their mature forms (such as the auditory and default mode networks) were found to be unilateral or incomplete. 


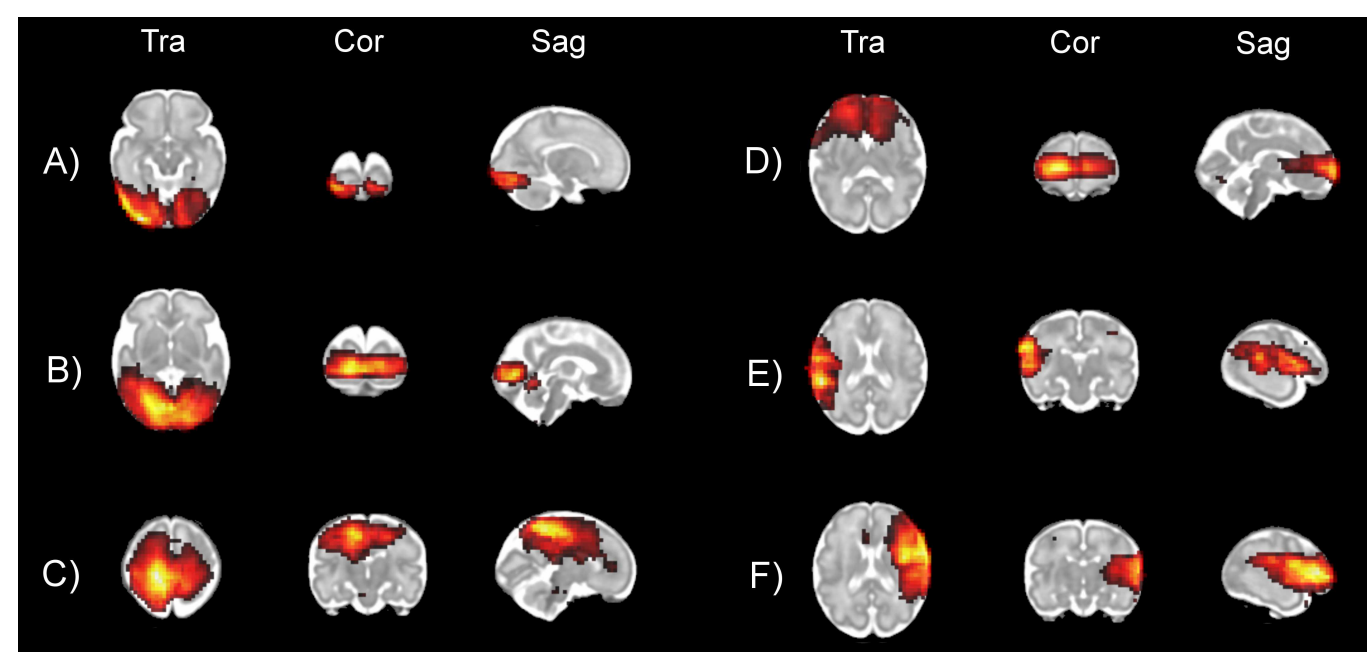

Figure 15: Transverse, coronal and sagittal views of RSNs given by Group

ICA overlaid onto a fetal template of 32 weeks (all z-score maps were thresholded using the cut-off value given by Melodic). The left side of the Figure corresponds to the left side of the brain.

\section{Discussion and Conclusions}

This paper proposes a framework to investigate spontaneous fluctuations of the BOLD signal in the human brain during a key period of development, before the natural term of birth. Performing fMRI of the fetus in utero requires methods that are tolerant of the large displacements caused both by maternal respiration and by unpredictable spontaneous movements by the fetus, leading to a challenging motion correction problem. Previous studies on fetuses employed methods directly adapted from those applied to adult data, in which complete stacks of slices covering the whole brain volume are processed together assuming negligible intra-stack movement. This assumption is frequently violated and results in a high rejection rate of data. The larger the fraction of the data that is excluded the greater the risk of bias. Such an approach is also inefficient in data usage, so loses statistical power. In this paper we have presented methods designed to directly address the consequences of motion with the aim of utilizing as much as possible of the acquired data.

The proposed method is a combined approach of slice to volume registration and scattered data interpolation with bias field and spin history corrections. 
The method was tested both with simulations and on in vivo data acquired from fetal subjects. Starting from raw stacks of EPI images acquired as parallel slices in scanner coordinates, it outputs data on a regular grid in an anatomical space in which the subject is static over the time course. These regular data are then available for any choice of fMRI analysis method. In this case, Group ICA was applied and was able to recover Resting State Networks that are consistent with those already mapped in preterm babies (Smyser et al., 2010; Doria et al., 2010) and have common features with RSNs found in older, term born neonates (Fransson et al., 2007, 2009).

The modular approach adopted in this study, in which motion correction is a separate step from functional data analysis offers great flexibility and is in line with most other processing pipelines currently adopted. It allowed us to focus on the key challenging feature of fetal datasets while providing results that are directly comparable with other analyses. A more integrated approach would be possible and there have been a number of studies in the adult fMRI literature that attempt to jointly address motion correction and functional analysis (Liao et al., 2005). A natural extension of the current approach for group analyses would be to project the scattered data directly into standard space and only then interpolate onto a standard regular grid. This is something we will explore in future studies.

The key enabling process in the proposed pipeline is alignment of each acquired slice within anatomical space $\left(\mathbf{S}_{2}\right)$. The simulations demonstrated that without this step the capability to detect functional signals is substantially reduced and there is supporting evidence from adult studies (Kim et al., 1999). Slice by slice alignment is well established for anatomical fetal imaging and proved effective in this application. However, there were three fetal subjects in the cohort for which we were not able to sufficiently correct for movement. The babies in question had moved rapidly for extended periods so that the earlier stages of the alignment processes, which rely on working with packages of temporally contiguous slices to robustly determine approximately correct slice locations, failed. Developing registration methods that can succeed with such chaotic data remains a challenge to be addressed further. A possible aid in this could be the adoption of multi-band acquisitions methods in which there is simultaneous acquisition of several slices (Larkman et al., 2001; Feinberg and Setsompop, 2013).

EPI images are extremely vulnerable to geometrical distortions caused by magnetic field inhomogeneity (Jezzard and Balaban, 1995). However, distortions in utero are much less pronounced than in other fMRI experiments 
for the simple reason that the fetal brain is surrounded by amniotic fluid and maternal soft tissue; tissue susceptibility differences at air-tissue boundaries are therefore generally not present close to the fetal head. For the data acquired at $1.5 \mathrm{~T}$ for this study, we found that distortion was not the first priority to be addressed and so focused on other challenges. The framework in its current form is however easily extendable, and does not prevent the inclusion of a distortion correction as preprocessing step.

Another failure mode for fetal fMRI occurs when the fetal head moves out of the prescribed imaging volume. Despite trying to enlarge the sampling volume to match the fetal brain size, this occurred in three cases in our sample. As increasing spatial coverage takes more time or requires less dense sampling in the slice selection direction, there is a tension between reducing the risk of data loss through large-scale changes in position and other key factors. In this study we adjusted the sampling density to enlarge the sampled volume in order to keep the other parameters matched across subjects. Fetal motion can cause gaps to open up between sampled slices in anatomical space, which is clearly sub-optimal and likely to be more severe with lower sampling density schemes. The focus in this study was to demonstrate a viable approach. It remains to be explored how data sampling density impacts on RSNs detection.

Notwithstanding the limitations discussed above, we were able to identify in the fetuses studied six RSNs with spatial representations consistent with those previously described in preterm infants. Networks that are likely to involve an underlying structure of long-range connectivity in full term neonatal subjects were found to be unilateral or incomplete in our fetal population, further supporting theories that their maturation is associated with rapid neuronal growth in the third trimester of human gestation (Doria et al., 2010). Because of the limited numbers of subjects in this pilot study, all were treated as a single group for the analysis, which means that a range of gestational ages and hence maturational states were combined. This will have increased the variability in the data, and the extracted networks are likely to emphasize the most conserved features, without sensitivity to detect emerging or rapidly changing features within the age range. Applying the proposed techniques to a larger study population will enable changes with gestational age to be explored. It can also equally be applied to experiments that seek to correlate fetal brain activity to external stimuli (Gowland and Fulford, 2004).

By fully correcting for fetal motion and the secondary effects of variable bias 
field and spin history, it is now possible to start to systematically perform fMRI in utero on a larger scale without the need to discard large fractions of data. The presented framework, which can be applied to resting state and stimulus based paradigms, holds promise as the basis for reliable and detailed functional brain mapping across the third trimester of gestation.

Acknowledgements.

GF thanks the Wellcome-EPSRC Medical Engineering Centre for a studentship. This research was supported by the MRC through a strategic research Grant (MR/K006355/1) and the EPSRC through programme grant $\mathrm{EP} / \mathrm{H} 046410 / 1$. 


\section{References}

Beckmann, C. F., Smith, S. M., 2004. Probabilistic independent component analysis for functional Magnetic Resonance Imaging. IEEE Transactions on Medical Imaging 23 (2), 137-152.

Bhagalia, R., Kim, B., 2008. Spin saturation artifact correction using slice-tovolume registration motion estimates for fMRI time series. Medical physics $35(2), 424-434$.

Biswal, B., Zerrin Yetkin, F., Haughton, V. M., Hyde, J. S., 1995. Functional connectivity in the motor cortex of resting human brain using echo-planar MRI. Magnetic resonance in medicine 34 (4), 537-541.

Buckner, R. L., Andrews-Hanna, J. R., Schacter, D. L., 2008. The brain's default network. Annals of the New York Academy of Sciences 1124 (1), $1-38$.

Calhoun, V. D., Liu, J., Adall, T., 2009. A review of group ICA for fMRI data and ICA for joint inference of imaging, genetic, and ERP data. Neuroimage 45 (1), S163-S172.

Damaraju, E., Phillips, J. R., Lowe, J. R., Ohls, R., Calhoun, V. D., Caprihan, A., 2010. Resting-state functional connectivity differences in premature children. Frontiers in systems neuroscience 4 (23), 1-13.

Damoiseaux, J., Rombouts, S., Barkhof, F., Scheltens, P., Stam, C., Smith, S. M., Beckmann, C., 2006. Consistent resting-state networks across healthy subjects. Proceedings of the National Academy of Sciences 103 (37), 13848-13853.

De Berg, M., Cheong, O., van Kreveld, M., Overmars, M., 2008. Computational geometry. Springer.

Delaunay, B. N., 1934. Sur la sphère vide. Bulletin of Academy of Sciences of the USSR 7 (6), 793-800.

Dempster, A., Laird, N., Rubin, D., 1977. Maximum likelihood from incomplete data via the EM-algorithm. Journal of the Royal Statistical Society 39 (1), 1-38. 
Doria, V., Beckmann, C. F., Arichi, T., Merchant, N., Groppo, M., Turkheimer, F. E., Counsell, S. J., Murgasova, M., Aljabar, P., Nunes, R. G., et al., 2010. Emergence of resting state networks in the preterm human brain. Proceedings of the National Academy of Sciences 107 (46), 20015-20020.

Duda, R., Hart, P., Stork, D., 2001. Pattern Classification., 2nd Edition. John Wiley \& Sons Inc.

Feinberg, D. A., Setsompop, K., 2013. Ultra-fast MRI of the human brain with simultaneous multi-slice imaging. Journal of Magnetic Resonance 229 (0), 90-100.

Fransson, P., Åden, U., Blennow, M., Lagercrantz, H., 2011. The functional architecture of the infant brain as revealed by resting-state fMRI. Cerebral Cortex 21 (1), 145-154.

Fransson, P., Skiöld, B., Engström, M., Hallberg, B., Mosskin, M., Åden, U., Lagercrantz, H., Blennow, M., 2009. Spontaneous brain activity in the newborn brain during natural sleep-an fMRI study in infants born at full term. Pediatric research 66 (3), 301-305.

Fransson, P., Skiöld, B., Horsch, S., Nordell, A., Blennow, M., Lagercrantz, H., Aden, U., 2007. Resting-state networks in the infant brain. Proceedings of the National Academy of Sciences 104 (39), 15531-15536.

Gao, W., Zhu, H., Giovanello, K. S., Smith, J. K., Shen, D., Gilmore, J. H., Lin, W., 2009. Evidence on the emergence of the brain's default network from 2-week-old to 2-year-old healthy pediatric subjects. Proceedings of the National Academy of Sciences 106 (16), 6790-6795.

Gowland, P., Fulford, J., 2004. Initial experiences of performing fetal fMRI. Experimental neurology 190 (1), 22-27.

Haacke, E. M., Brown, R. W., Thompson, M. R., Venkatesan, R., 1999. Magnetic resonance imaging: physical principles and sequence design. Vol. 1. Wiley-Liss New York:.

Hayat, T., Nihat, A., Martinez-Biarge, M., McGuinness, A., Allsop, J., Hajnal, J., Rutherford, M., 2011. Optimization and initial experience of a multisection balanced steady-state free precession cine sequence for the 
assessment of fetal behavior in utero. American Journal of Neuroradiology $32(2), 331-338$.

Hyvärinen, A., Oja, E., 2000. Independent component analysis: algorithms and applications. Neural networks 13 (4), 411-430.

Jezzard, P., Balaban, R. S., 1995. Correction for geometric distortion in echo planar images from B0 field variations. Magnetic resonance in medicine $34(1), 65-73$.

Jiang, S., Xue, H., Glover, A., Rutherford, M., Rueckert, D., Hajnal, J. V., 2007. MRI of moving subjects using multislice snapshot images with volume reconstruction (SVR): application to fetal, neonatal, and adult brain studies. IEEE Transactions on Medical Imaging 26 (7), 967-980.

Juntu, J., Sijbers, J., Van Dyck, D., Gielen, J., 2005. Bias field correction for mri images. In: Computer Recognition Systems. Vol. 30. Springer, pp. $543-551$.

Kim, B., Boes, J. L., Bland, P. H., Chenevert, T. L., Meyer, C. R., 1999. Motion correction in fMRI via registration of individual slices into an anatomical volume. Magnetic resonance in medicine 41 (5), 964-972.

Kim, K., Habas, P. A., Rajagopalan, V., Scott, J. A., Corbett-Detig, J. M., Rousseau, F., Barkovich, A. J., Glenn, O. A., Studholme, C., 2011. Bias field inconsistency correction of motion-scattered multislice MRI for improved 3D image reconstruction. Medical Imaging, IEEE Transactions on 30 (9), 1704-1712.

Kuklisova-Murgasova, M., Cifor, A., Napolitano, R., Papageorghiou, A., Quaghebeur, G., Rutherford, M. A., Hajnal, J. V., Noble, J. A., Schnabel, J. A., 2013. Registration of 3D fetal neurosonography and MRI. Medical Image Analysis 17 (8), 1137-1150.

Kuklisova-Murgasova, M., Quaghebeur, G., Rutherford, M. A., Hajnal, J. V., Schnabel, J. A., 2012. Reconstruction of fetal brain MRI with intensity matching and complete outlier removal. Medical Image Analysis 16 (8), $1550-1564$.

Larkman, D. J., Hajnal, J. V., Herlihy, A. H., Coutts, G. A., Young, I. R., Ehnholm, G., 2001. Use of multicoil arrays for separation of signal from 
multiple slices simultaneously excited. Journal of Magnetic Resonance Imaging 13 (2), 313-317.

Lee, S., Wolberg, G., Shin, S. Y., 1997. Scattered data interpolation with multilevel b-splines. Visualization and Computer Graphics, IEEE Transactions on 3 (3), 228-244.

Liao, R., Krolik, J. L., McKeown, M. J., 2005. An information-theoretic criterion for intrasubject alignment of FMRI time series: motion corrected independent component analysis. Medical Imaging, IEEE Transactions on 24 (1), 29-44.

Malamateniou, C., Malik, S., Counsell, S., Allsop, J., McGuinness, A., Hayat, T., Broadhouse, K., Nunes, R., Ederies, A., Hajnal, J., et al., 2013. Motioncompensation techniques in neonatal and fetal MR imaging. American Journal of Neuroradiology 34 (6), 1124-1136.

Rousseau, F., Glenn, O. A., Iordanova, B., Rodriguez-Carranza, C., Vigneron, D. B., Barkovich, J. A., Studholme, C., 2006. Registration-based approach for reconstruction of high-resolution in utero fetal MR brain images. Academic radiology 13 (9), 1072-1081.

Schöpf, V., Kasprian, G., Brugger, P., Prayer, D., 2012. Watching the fetal brain at "rest". International Journal of Developmental Neuroscience 30 (1), 11-17.

Serag, A., Kyriakopoulou, V., Rutherford, M. A., Edwards, A. D., Hajnal, J. V., 2012. A multi-channel 4D probabilistic atlas of the developing brain: Application to fetuses and neonates. Annals of the BMVA 2012 (3), 1-14.

Sled, J. G., Zijdenbos, A. P., Evans, A. C., 1998. A nonparametric method for automatic correction of intensity nonuniformity in MRI data. Medical Imaging, IEEE Transactions on 17 (1), 87-97.

Smyser, C. D., Inder, T. E., Shimony, J. S., Hill, J. E., Degnan, A. J., Snyder, A. Z., Neil, J. J., 2010. Longitudinal analysis of neural network development in preterm infants. Cerebral Cortex 20 (12), 2852-2862.

Styner, M., Brechbuhler, C., Szckely, G., Gerig, G., 2000. Parametric estimate of intensity inhomogeneities applied to MRI. Medical Imaging, IEEE Transactions on 19 (3), 153-165. 
Thomason, M. E., Dassanayake, M. T., Shen, S., Katkuri, Y., Alexis, M., Anderson, A. L., Yeo, L., Mody, S., Hernandez-Andrade, E., Hassan, S. S., et al., 2013. Cross-hemispheric functional connectivity in the human fetal brain. Science translational medicine 5 (173), 1-10.

Van Den Heuvel, M. P., Hulshoff Pol, H. E., 2010. Exploring the brain network: a review on resting-state fMRI functional connectivity. European Neuropsychopharmacology 20 (8), 519-534.

Vovk, U., Pernus, F., Likar, B., 2007. A review of methods for correction of intensity inhomogeneity in MRI. Medical Imaging, IEEE Transactions on 26 (3), 405-421.

Wells III, W. M., Grimson, W. E. L., Kikinis, R., Jolesz, F. A., 1996. Adaptive segmentation of MRI data. IEEE Transactions on Medical Imaging 15 (4), 429-442.

Williams, L.-A., Gelman, N., Picot, P. A., Lee, D. S., Ewing, J. R., Han, V. K., Thompson, R. T., 2005. Neonatal brain: Regional variability of in vivo MR imaging relaxation rates at $3.0 \mathrm{~T}$ - initial experience. Radiology 235 (2), 595-603.

Yancey, S. E., Rotenberg, D. J., Tam, F., Chiew, M., Ranieri, S., Biswas, L., Anderson, K. J., Baker, S. N., Wright, G. A., Graham, S. J., 2011. Spinhistory artifact during functional MRI: Potential for adaptive correction. Medical physics 38 (8), 4634-4646. 